\title{
Efficient global optimization of reservoir geomechanical parameters based on synthetic aperture radar-derived ground displacements
}

\author{
Francesco Comola ${ }^{1}$, Carlo Janna ${ }^{2}$, Alberto Lovison ${ }^{3}$, Marco Minini ${ }^{4}$, \\ Andrea Tamburini ${ }^{4}$, and Pietro Teatini ${ }^{2}$
}

\begin{abstract}
When large volumes of fluids are removed from or injected into underground formations for, e.g., hydrocarbon and water production, $\mathrm{CO}_{2}$ storage, gas storage, and geothermal energy exploitation, monitoring of surface deformations coupled to numerical modeling improves our understanding of reservoir behavior. The ability to accurately simulate surface displacements, however, is often impaired by limited information on reservoir geometry, waterdrive strength, and fluid-geomechanical parameters characterizing the geologic formations of interest. We have investigated the ability of efficient global optimization (EGO) to reduce the parameter uncertainties usually affecting geomechanical modeling. EGO is used to identify the parameter set that minimizes the difference in land displacements obtained from synthetic aperture radar (SAR)-derived measurements and $3 \mathrm{D}$ geomechanical modeling. We have tested the approach on
\end{abstract}

the Tengiz giant oil field, Kazakhstan, where large uncertainties affect our knowledge of geomechanical parameters and pore pressure evolution. SqueeSAR on ENVISAT and RADARSAT-1 images acquired between 2004 and 2007 provided a set of high-precision, high-areal-density subsidence measurements of the test site. Based on the available information, a 3D geomechanical model of the reservoir has been developed using the elastoplastic finite-element code GEPS3D. Our results indicated that EGO efficiently identifies the global optimum in the parameter space, yielding a significant reduction in the difference between modeled and measured land subsidence. The match between simulated and SAR-measured horizontal displacements was developed as validation of the EGO calibration, which thus proved an effective and rather inexpensive method for the simultaneous management of several uncertainties and the reliable quantification of the rock properties.

\section{INTRODUCTION}

It is widely recognized that anthropogenic activities involving the extraction or the injection of fluids from or into the subsurface cause a change in the original pore pressure. The consequent adjustment of the stress field and the volumetric deformation of the geologic formations can reactivate faults located in the surroundings of the reservoir (Trugman et al., 2014), induce seismic events (Lei et al., 2013), produce localized ground ruptures ( $\mathrm{Li}$ et al., 2000), land subsidence (Fielding et al., 1998; Motagh et al., 2008; Chang et al.,
2014), or uplift (Vasco et al., 2010; Teatini et al., 2011). Thus, reservoir management raises serious concerns in terms of human health, safety of structures and infrastructures, economic risks, and environmental and hydrologic impact (Morton et al., 2006). The probability of these processes is largely influenced by the geomechanical properties of the reservoir and the surrounding geologic horizons.

The state of the art in monitoring the stress/strain field at the reservoir depth has advanced, owing to new instrumentation and sensors such as the radioactive marker technique (Ferronato et al., 2003), time-lapse seismic methods (Alassi et al., 2010), and micro-

\footnotetext{
Manuscript received by the Editor 27 July 2015; revised manuscript received 22 December 2015; published online 20 April 2016.

${ }_{1}^{1}$ School of Architecture, Civil and Environmental Engineering, École Polytechnique Fédérale de Lausanne, Lausanne, Switzerland and M ${ }^{3}$ E S.r.l., Padova, Italy. E-mail: francesco.comola@gmail.com.

${ }^{2}$ University of Padova, Department of Civil, Environmental and Architectural Engineering, Padova, Italy and M³ E S.r.l., Padova, Italy. E-mail: carlo.janna@ unipd.it; pietro.teatini@unipd.it.

${ }^{3}$ University of Padova, Department of Mathematics, Padova, Italy and $\mathrm{M}^{3} \mathrm{E}$ S.r.1., Padova, Italy. E-mail: lovison@math.unipd.it.

${ }^{4}$ Tele-Rilevamento Europa, Milano, Italy. E-mail: marco.minini@treuropa.com; andrea.tamburini@ treuropa.com.

(C) 2016 Society of Exploration Geophysicists. All rights reserved.
} 
seismic monitoring (Maxwell et al., 2010). Despite these technological advances, measurements of ground surface displacements, caused by stress rearrangements at depth, still represent the largest and most comprehensive data set available to characterize the geomechanical behavior of the system. The measure of land motions above producing reservoirs has advanced immensely over the past two decades owing to the development of synthetic aperture radar (SAR)-based methodologies (Fielding et al., 1998; Xu et al., 2001). The most recent multi-image multitrack technologies (Hooper, 2008; Ferretti et al., 2011) provide high-precision (millimetric) time series of vertical and horizontal west-east displacements on highdensity (up to a few thousands per $\mathrm{km}^{2}$ ) coherent radar reflectors spread on the study area.

Numerical modeling represents a unique approach for a simultaneous and coherent interpretation of deep data and surface information. Advanced 3D viscoelastoplastic models have been used to reproduce the geomechanical behavior of complex, multipool, faulted reservoirs worldwide (Ferronato et al., 2003; Vasco et al., 2010; Teatini et al., 2011; Castelletto et al., 2013; Rinaldi and Rutqvist, 2013). Despite significative developments, model reliability in predicting localized (e.g., along fault systems) and/or distributed (e.g., land subsidence) deformations of subsurface systems due to change in pore pressure is hampered by large uncertainties in model parameters. On one side, data coverage is often too limited to allow spatially distributed parameter estimations. On the other side, the temporal sampling of time-lapse geophysics surveys can be too sparse to capture important transient phenomena. Moreover, laboratory tests used to characterize small-scale geomechanical properties of deep samples are not usually representative at the reservoir scale, where geologic features such as fractures and faults control flow and deformation.

The identification of model parameters based on observed data is known as the inverse problem, and the related solution procedure is often referred to as model calibration. The inverse approaches com-

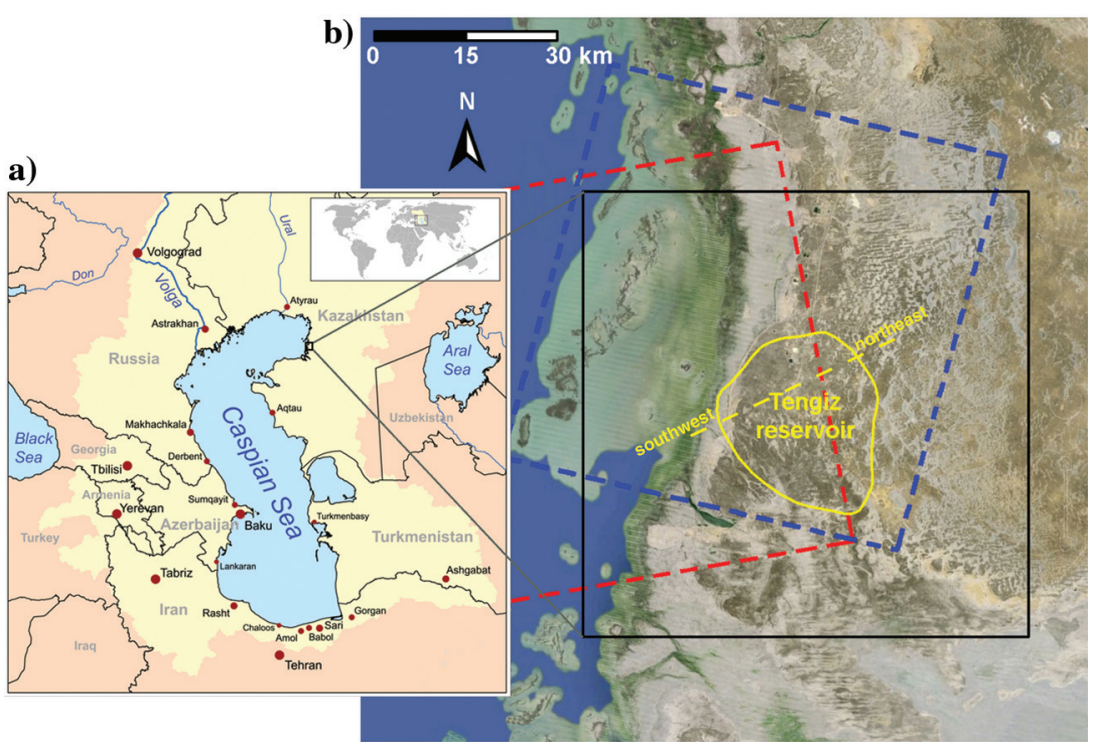

Figure 1. (a) Location of the pre-Caspian basin. (b) Trace of the Tengiz giant oil reservoir on the coastland of the northern Caspian Sea, Kazakhstan. The black box represents the areal extent of the geomechanical model, the dashed red and blue squares represent the RADARSAT-1 and ENVISAT track, respectively, used to measure the movement of the ground surface. monly used in geomechanics present several shortcomings. For instance, gradient-based methods (Oreste, 2005) may converge to local minima if the objective function is not convex, whereas genetic or other evolutionary algorithms (Levasseur et al., 2008) do not hinge on exact convergence properties and are usually computationally expensive. Only recently have appropriate inversion methodologies such as inversion algorithms (Iglesias and McLaughlin, 2012; Hesse and Stadler, 2014) and data assimilation techniques (Chang et al., 2010; Baù et al., 2015; Zoccarato et al., 2016) been implemented to resolve, or at least reduce, uncertainty problems in geomechanics. However, the simultaneous management of several uncertain parameters is still a challenge for most inverse approaches (Chang et al., 2010) and often leads to nonphysical solutions and underdetermined or otherwise ill-posed problems (Oliver and Chen, 2011).

For problems of relatively large dimension (up to 10 uncertain parameters), the forward efficient global optimization (EGO) approach (Jones et al., 1998) has proven a very efficient procedure, becoming largely used for mechanical engineering applications in recent years (Jones, 2001; Wang and Shan, 2007; Lovison and Rigoni, 2010). Owing to exact convergence properties and small computational burden, EGO has the potential to be an effective optimization method also for geomechanical applications. Therefore, our objective is to investigate the ability of EGO to explore the geomechanical system response to uncertain parameters and provide the optimal solution. Here, we test EGO in a geomechanical context for the first time, with an application to the case study of the Tengiz giant oil field, Kazakhstan (Figure 1).

\section{METHODOLOGY}

This section provides a detailed description of all the methods applied during the simulation-optimization procedure, i.e., the geomechanical model, SqueeSAR technology, and EGO. For an explanation of how these techniques are combined to perform the model calibration, the reader is referred to the "Simulation-optimization procedure" section.

\section{Geomechanical model}

Injection or extraction of fluids from underground produces a pore-pressure change that is responsible for deformations at depth, which may propagate up to the ground surface. The mechanism of this process is described by the Biot principle (Biot, 1941) that separates the total stress acting on a porous volume into the sum of the effective stress, i.e., the stress acting between rock grains, and the fluid pressure. In vectorial form, this relationship is expressed as

$$
\overline{\boldsymbol{\sigma}}=\boldsymbol{\sigma}-\alpha p \mathbf{i},
$$

where $\overline{\boldsymbol{\sigma}}^{T}=\left[\bar{\sigma}_{x}, \bar{\sigma}_{y}, \bar{\sigma}_{z}, \bar{\tau}_{x y}, \bar{\tau}_{y z}, \bar{\tau}_{z x}\right]$ is the total stress in the $x$ (west-east), $y$ (south-north), and $z$ (vertical) reference frame, $\boldsymbol{\sigma}^{T}=\left[\sigma_{x}, \sigma_{y}, \sigma_{z}, \tau_{x y}, \tau_{y z}, \tau_{z x}\right]$ is the effective stress, $p$ is the fluid pressure, $\mathbf{i}=[1,1,1,0,0,0]$ is the Kronecker $\delta$, and $\alpha$ is the Biot coefficient. 
The porous volume deformation can be found by applying the virtual work theorem

$$
\int_{\Omega} \boldsymbol{\epsilon}^{v, T}(\boldsymbol{\sigma}-\alpha p \mathbf{i}) d \Omega=0,
$$

where inertial forces are neglected, null external forces are assumed, and $\boldsymbol{\epsilon}^{v}$ denotes virtual deformations. The effective stress $\boldsymbol{\sigma}$ in equation 2 depends on real deformation $\boldsymbol{\epsilon}$ through a constitutive relationship

$$
\boldsymbol{\sigma}=\boldsymbol{\sigma}(\boldsymbol{\epsilon})
$$

which strongly depends on the mechanical properties and stress history of the formation of interest. The deformed configuration of the porous medium is found by applying to equation 2 a classical finite-element (FE) discretization of the unknown displacements (Zienkiewicz and Taylor, 2000) and imposing appropriate boundary conditions.

In this analysis, the stress-strain relationship plays a central role as most of the uncertainties stem from the mechanical properties of the deep rocks. In geomechanics, the stress-strain relationship is usually expressed in incremental form

$$
d \boldsymbol{\sigma}=\mathbf{D} d \boldsymbol{\epsilon},
$$

where $\mathbf{D}$ is the tangent constitutive matrix. Assuming a mechanically isotropic medium, the strain-stress relation reads

$$
\begin{aligned}
\left\{\begin{array}{l}
d \sigma_{x x} \\
d \sigma_{y y} \\
d \sigma_{z z} \\
d \tau_{x y} \\
d \tau_{y z} \\
d \tau_{z x}
\end{array}\right\}=\frac{E(1-\nu)}{(1+\nu)(1-2 \nu)} \\
\times\left[\begin{array}{cccccc}
1 & \frac{\nu}{1-\nu} & \frac{\nu}{1-\nu} & 0 & 0 & 0 \\
\frac{\nu}{1-\nu} & 1 & \frac{\nu}{1-\nu} & 0 & 0 & 0 \\
\frac{\nu}{1-\nu} & \frac{\nu}{1-\nu} & 1 & 0 & 0 & 0 \\
0 & 0 & 0 & \frac{1-2 \nu}{2(1-\nu)} & 0 & 0 \\
0 & 0 & 0 & 0 & \frac{1-2 \nu}{2(1-\nu)} & 0 \\
0 & 0 & 0 & 0 & 0 & \frac{1-2 \nu}{2(1-\nu)}
\end{array}\right]\left\{\begin{array}{l}
d \epsilon_{x x} \\
d \epsilon_{y y} \\
d \epsilon_{z z} \\
d \gamma_{x y} \\
d \gamma_{y z} \\
d \gamma_{z x}
\end{array}\right\},
\end{aligned}
$$

where $E$ and $\nu$ are the Young modulus and Poisson's ratio, respectively. The coefficient $E(1-\nu) /(1+\nu)(1-2 \nu)$ in equation 5, which represents the ratio between an external vertical load and the relative compaction $(\Delta h / h)$ of a rock sample loaded in oedometric conditions, is the inverse of the vertical compressibility $c_{M}$

$$
c_{M} \equiv \frac{(1+\nu)(1-2 \nu)}{E(1-\nu)} .
$$

The geomechanical response of a reservoir is investigated by a one-way coupling approach in which the pore-pressure changes are imposed as external loads in the geomechanical simulator. Equation 2 is solved numerically by FEs using the code GEPS3D (Geomechanical Elasto-Plastic 3D Simulator developed at the University of Padova), which follows the infinite pore pressure gradient formulation (Gambolati et al., 2001). GEPS3D has been successfully used in several geomechanical applications over the past few years (Teatini et al., 2000, 2011; Ferronato et al., 2003; Janna et al., 2012; Castelletto et al., 2013). An advanced block factored sparse approximate inverse with incomplete LU factorization preconditioner (Janna et al., 2010) is implemented in GEPS3D to effectively solve on parallel supercomputers the typically large linear systems arising from FE implementation.

\section{SqueeSAR}

SqueeSAR technology (Ferretti et al., 2011) provides a significant number of ground displacement measurements by exploiting radar sensors mounted on specific satellites. Radar signals are reflected by selected measurement points (MPs), which can be either permanent scatterers (PSs) or distributed scatterers (DSs), on the earth surface. Provided enough (at least 15-20) SAR images are available over the area of interest, the following information can be retrieved: geographic coordinates (latitude, longitude, and elevation), average annual velocity, and the time series of ground displacement of the MP. All the displacement measurements are projections of the displacement vector along the satellite line of sight (LOS). SqueeSAR results obtained from the processing of ascending and descending satellite orbits can be combined to give separate estimates of vertical and west-east movements. When two estimates of the same MP velocity are available along ascending and descending directions, $V_{a}$ and $V_{d}$, respectively, the velocity of an MP can be expressed in the Cartesian reference system $x-y-z$ as

$$
\mathbf{V}=V_{x} \mathbf{s}_{x}+V_{y} \mathbf{s}_{y}+V_{z} \mathbf{s}_{z},
$$

where $V_{x}, V_{y}$, and $V_{z}$ represent the component of the velocity $\mathbf{V}$ along the west-east, north-south, and vertical directions, and $\mathbf{s}_{x}, \mathbf{s}_{y}$, and $\mathbf{s}_{z}$ are the standard basis vectors of the coordinate system. Because the satellite orbit is known, the LOS with respect to the $x-y-z$ coordinate system and the corresponding direction cosines of the velocity vector $V_{a}$ and $V_{d}$ can be determined. The following system can be written:

$$
\begin{aligned}
& V_{a}=V_{x} s_{x, a}+V_{y} s_{y, a}+V_{z} s_{z, a}, \\
& V_{d}=V_{x} s_{x, d}+V_{y} s_{y, d}+V_{z} s_{z, d},
\end{aligned}
$$

where $s_{x, a}, s_{y, a}, s_{z, a}$ and $s_{x, d}, s_{y, d}, s_{z, d}$ represent the direction cosines of the velocity vectors $V_{a}$ and $V_{d}$, measured along the satellite LOS, and the west-east, north-south, and vertical directions, respectively. The previous system represents an ill-posed problem because it has three unknowns $\left(V_{x}, V_{y}\right.$, and $\left.V_{z}\right)$ and only two equations. However, because the satellite orbit is substantially parallel to the meridian, the LOS sensitivity to possible motion in the north-south direction is small $\left(s_{y, a} \simeq 0\right.$ and $s_{y, d} \simeq 0$ ). Rewriting equations 8 and 9 with this approximation and solving for $V_{x}$ and $V_{z}$ yields

$$
\begin{gathered}
V_{x}=\frac{V_{a} s_{z, d}-V_{d} s_{z, a}}{s_{x, a} s_{z, d}-s_{z, a} s_{x, d}}, \\
V_{z}=\frac{V_{d} s_{x, a}-V_{a} s_{x, d}}{s_{x, a} s_{z, d}-s_{z, a} s_{x, d}} .
\end{gathered}
$$


Given that the MP of the ascending and descending data sets does not generally correspond to the same physical objects, common targets must be selected following geographical considerations. Namely, the area of interest is divided into $50 \times 50 \mathrm{~m}$ square cells and, in each of them, the velocities and time series of all scatterers are averaged under the hypothesis that they exhibit a similar displacement. This operation is applied to the ascending and descending data sets, resulting in a regularization of the spatial distribution of the two data sets on a common grid. At this stage, cells that contain a target in the ascending and descending data sets are used to estimate the vertical and west-east motions. A trivial integration in time of $V_{x}$ and $V_{z}$ provides the average displacement of each MP over the period of interest. Moreover, SqueeSAR provides as a secondary result the (possibly nonlinear) movement time series of each MP with respect to the first processed SAR image.

\section{Efficient global optimization}

The EGO strategy (Jones et al., 1998) consists of an iterative procedure for detecting the global optimum of a loss function defined in a bounded multidimensional domain. In this geomechanical application, the loss function measures the difference between the ground displacements computed by the geomechanical model and those provided by the SAR technology. Accordingly, the global optimum is the set of $M$ uncertain model parameters that minimizes the loss function in an $M$-dimensional domain, whose bounds are defined a priori by assigning proper ranges of variation to the optimization parameters. Let us denote with $\eta_{\mathbf{m}}$ the SAR-derived displacement rates and with $\eta_{\mathbf{c}}$ the computed displacement rates obtained with an $M$-dimensional set $\mathbf{c}$ of parameters. The loss function $L(\mathbf{c})$ is therefore defined as

$$
L(\mathbf{c})=-\frac{1}{\sqrt{\sum_{i}\left(\eta_{\mathbf{m}, i}-\eta_{\mathbf{c}, i}\right)^{2}}} .
$$

The summation in equation 12 is extended over the total number of MPs, in which the numerical solution is interpolated based on the nodal values of the FE grid. The only necessary assumption for EGO is the Lipschitz regularity of the loss function, i.e., the slope of the line connecting each couple of points on the function's graph must be finite. Even though the features of the loss function are not known a priori, Lipschitz regularity is a rather mild condition very frequently met in optimization problems.

Here, $L(\mathbf{c})$ is initially computed in a starting sample $\mathbf{c}_{N}$, formed of a number $N$ of parameter sets, identified with a Latin hypercube design (LHD) (see, e.g., Lovison and Rigoni, 2010), to grossly yet uniformly explore the function domain. The LHD adopts a uniform distribution and does not account for correlations among parameters.

The EGO iteration consists of identifying the parameter set $\mathbf{c}^{*}$ providing the maximum value of an auxiliary Gaussian covariance-based function $\mathcal{E}$ called expected improvement (Jones, 2001), which measures the probability that $L(\mathbf{c})$, for an untried parameter set $\mathbf{c}$, is smaller than the minimum value of the loss function in the starting sample, i.e., $L_{\min }=\min _{N} L\left(\mathbf{c}_{N}\right)$. For any given untried set c, a Gaussian process model is used to produce a predictive distribution

$$
\tilde{L}(\mathbf{c})=\mathcal{N}(\mu(\mathbf{c}), \sigma(\mathbf{c})),
$$

where the prediction of the loss function at a generic site $\mathbf{c}$ is a normal probability distribution with mean $\mu(\mathbf{c})$ and variance $\sigma^{2}(\mathbf{c})$. Estima- tions for mean and variance are provided by a kriging metamodel $\mathbf{c} \mapsto K_{0}\left(\mathbf{c} ; \mathbf{c}_{N}\right)$, i.e., a statistical interpolation performed on the basis of the starting sample $\mathbf{c}_{N}$.

The improvement $I(\mathbf{c})$ is also a random variable given as

$$
I(\mathbf{c})=\max \left(L_{\min }-\tilde{L}(\mathbf{c}), 0\right),
$$

thus the expected improvement is

$$
\mathcal{E}[I(\mathbf{c})]=\mathcal{E}\left[\max \left(L_{\min }-\tilde{L}(\mathbf{c}), 0\right)\right]
$$

If we denote with $\phi$ the probability density function of the standard normal distribution, and with $\Phi$ its cumulative distribution function, the expected improvement can be expressed in closed form as (Jones et al., 1998)

$$
\begin{aligned}
\mathcal{E}[I(\mathbf{c})]= & \left(L_{\min }-\mu(\mathbf{c})\right) \Phi\left(\frac{L_{\min }-\mu(\mathbf{c})}{\sigma(\mathbf{c})}\right) \\
& +\sigma(\mathbf{c}) \phi\left(\frac{L_{\min }-\mu(\mathbf{c})}{\sigma(\mathbf{c})}\right) .
\end{aligned}
$$

The existence of a closed form allows us to exploit the structure of $\mathcal{E}[I(\mathbf{c})]$ to find its maximum. The computation of the maximum expected improvement is greatly facilitated by the fact that $\mathcal{E}[I(\mathbf{c})]$ is monotonically decreasing with $\mu(\mathbf{c})$ and monotonically increasing with $\sigma(\mathbf{c})$. In fact, a straightforward computation of the derivatives in equation 16 gives (Jones et al., 1998)

$$
\begin{gathered}
\frac{\partial \mathcal{E}}{\partial \mu}=-\Phi\left(\frac{L_{\min }-\mu(\mathbf{c})}{\sigma(\mathbf{c})}\right)<0, \\
\frac{\partial \mathcal{E}}{\partial \sigma}=\phi\left(\frac{L_{\min }-\mu(\mathbf{c})}{\sigma(\mathbf{c})}\right)>0,
\end{gathered}
$$

where the above-mentioned equations suggest that $\mathcal{E}$ is large not only in regions of the $M$-dimensional domain where $\mu(\mathbf{c})$ is low, i.e., where the loss value is likely to be small, but also where $\sigma(\mathbf{c})$ is large, i.e., where the sampling density is low and the uncertainty on the loss value is large.

Before a new EGO iteration is carried out, $L\left(\mathbf{c}^{*}\right)$ is evaluated by rerunning the numerical model and the tested set $\mathbf{c}^{*}$ is added to the starting sample $\mathbf{c}_{N+1}$. Iteratively, the size of the starting sample increases and EGO is provided with more and more spatially distributed information on the loss function to update the kriging metamodel and seek new candidates $\mathbf{c}^{*}$.

By pursuing a maximization of the expected improvement, EGO iteratively scouts neighborhoods of current optima and, occasionally, poorly sampled regions. This explains how EGO balances the merits of local and global optimization algorithms by performing an efficient search and avoiding the risk of being stuck in local minima. Under nondegeneracy conditions (for more technical details, see, e.g., Bull, 2011), it is possible to prove that unexplored regions, which have large $\sigma(\mathbf{c})$, will be sooner or later sampled by maximizing the expected improvement. Therefore, the domain is densely sampled everywhere in infinite time and global convergence is guaranteed (Jones, 2001; Kleijnen et al., 2012). Global convergence cannot be achieved in a finite number of evaluations unless additional information on the regularity of the problem is available, e.g., a global Lipschtiz constant for the loss function. Even though such 
information is generally not available, it is still reasonable to assume that the loss function has some level of regularity. Therefore, unexplored regions are not likely to hide the global optimum when the number of evaluation grows, because this would imply higher and higher Lipschitz constants and a decreasing level of regularity. Hence, from a practical point of view, the experimenter should combine his/her own knowledge on the problem regularity, coming from his/her experience and physical considerations, to decide if the algorithm has explored densely enough or with the required accuracy the ranges of the parameters. Theoretical considerations and plenty of benchmarks documented in the literature suggest that if the loss function is smooth enough the method provides a good approximation of the global optimum in a relatively small number of iterations.

\section{CASE STUDY}

The Tengiz reservoir is made by a large build-up of limestone, essentially a large atoll built on the margin of the deep pre-Caspian basin, northern Caspian Sea, Kazakhstan (Figure 1). The atoll was built by marine organisms mostly during Carboniferous time and is sealed by thick, impermeable Kungurian salt of Permian age. The Tengiz oil field is $19 \mathrm{~km}$ wide and $21 \mathrm{~km}$ long and spans the depth range $z$ between 4300 and $5500 \mathrm{~m}$ below the mean sea level.

The reservoir geometry presents a central platform, where the majority of the wells are placed, an outer platform, and a slope as shown in Figure 2. Discovered in 1979, this reservoir had recoverable reserves estimated between six and nine billion barrels of oil and is still one of the largest discoveries in recent history.

Although the reservoir geometry and fluid-dynamic properties are sufficiently known from a few papers (Weber et al., 2003; Collins et al., 2006), little information is available on the time and space evolution of the pore pressure. The oil was highly overpressured at 830 bar $(83 \mathrm{MPa})$ when the field was first tapped and, in 2007, the pressure decreased to approximately $550 \mathrm{bar}(55 \mathrm{MPa})$ in the central platform and to $620-750$ bar $(62-75 \mathrm{MPa})$ in the remaining portions. The pressure decreased almost uniformly and linearly with

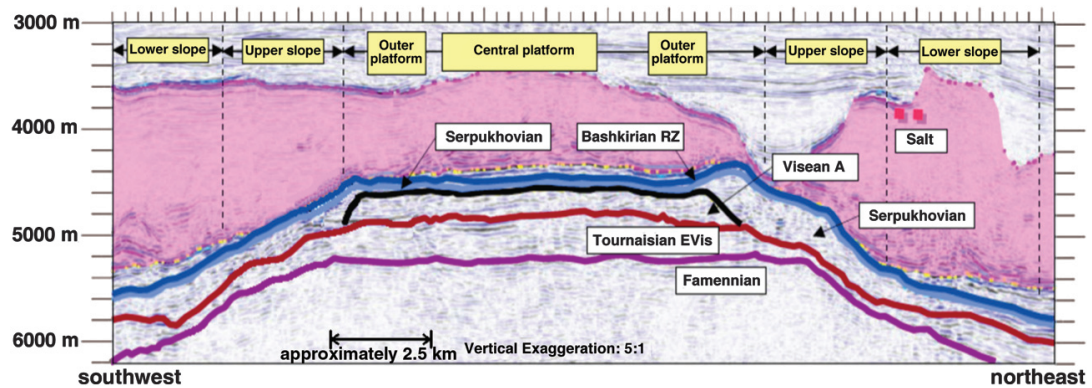

Figure 2. Geologic section of the reservoir geometry along the southwest-northeast alignment shown in Figure $1 \mathrm{~b}$. The dark blue line indicates the interface between the reservoir and the overburden. The maximum reservoir depth is estimated at $5500 \mathrm{~m}$ depth (modified after Collins et al., 2006)

a)

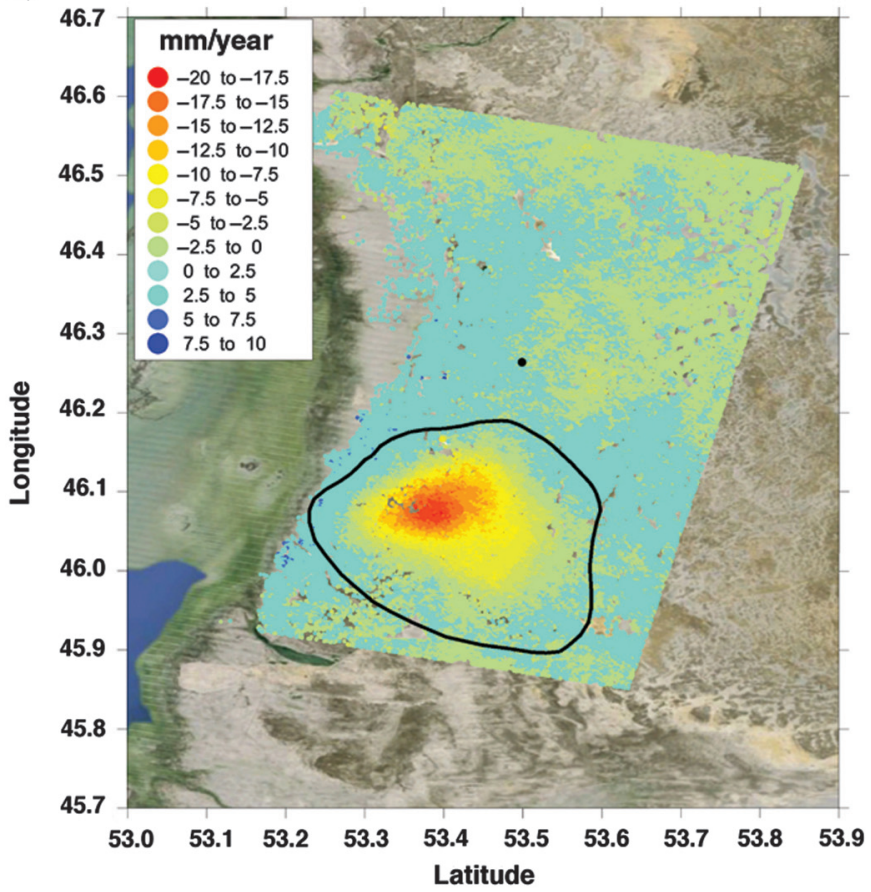

b)

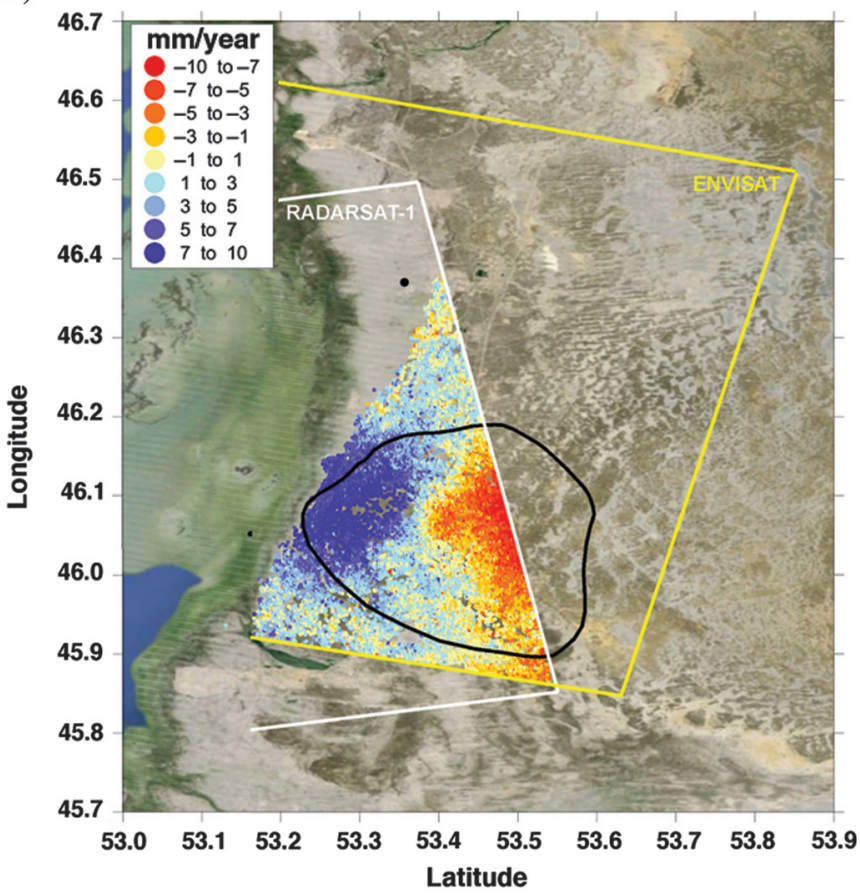

Figure 3. Average land displacements (mm/year) measured by SqueeSAR above the coastland of the northern Caspian Sea, Kazakhstan, from 2004 to 2007. (a) LOS displacement rates from ENVISAT images and (b) west-east displacement rates obtained by combining ascending ENVISAT and descending RADARSAT-1 images. Negative values mean a moving away from the satellite in panel (a), that is substantially land subsidence, and westward displacements in panel (b). The black dot and solid line represent the reference of the SAR solution and the trace of the Tengiz giant oil reservoir, respectively. The RADARSAT-1 and ENVISAT track used to measure the movement of the ground surface are shown in panel (b) by a white and yellow box, respectively. 
time in the platform region. Conversely, pressure change is largely uneven in the slopes, which are significantly fractured (Dagistanova et al., 2011). The geomechanical properties of the reservoir and the surrounding basin are also uncertain. Available information collected from unpublished reports and reference books on the physical properties of rocks (Carmichael, 1989) provides the following ranges for the uniaxial vertical compressibility $c_{M}$ and Poisson's ratio $\nu$ : $6 \times 10^{-6}<c_{M}<6 \times 10^{-5} \mathrm{bar}^{-1}$ and $0.30<\nu<0.35$ in the allu$\mathrm{vial} / \mathrm{salt}$ overburden/sideburden $(0<z<5500 \mathrm{~m}), 2 \times 10^{-6}<c_{M}<$ $6 \times 10^{-6} \mathrm{bar}^{-1}$ and $0.20<\nu<0.27$ in the carbonate reservoir $(4300<z<5500 \mathrm{~m})$, and $c_{M} \simeq 2 \times 10^{-6} \mathrm{bar}^{-1}$ and $\nu \simeq 0.32$ in the clastic underburden $(z>5500 \mathrm{~m})$.

The information on the geometry of the Tengiz oil field is used to generate a tetrahedral FE mesh for geomechanical modeling. The model domain covers an areal extent of $80 \times 80 \mathrm{~km}$ centered on the reservoir and is confined above by the ground surface and below by a fixed basement at a $10 \mathrm{~km}$ depth. Standard conditions with zero displacement on the outer and bottom boundaries are prescribed, whereas the land surface is a no-stress boundary. The ground surface does not present any relevant topographical features and is assumed horizontal in the model. The domain is discretized into 548540 tetrahedra with 95,508 nodes.

Several 19 ENVISAT descending scenes and 32 RADARSAT-1 ascending scenes acquired between 2004 and 2007 over the Tengiz reservoir are processed by SqueeSAR. The PS/DS selection is carried out by setting to 0.8 the minimum temporal coherence,

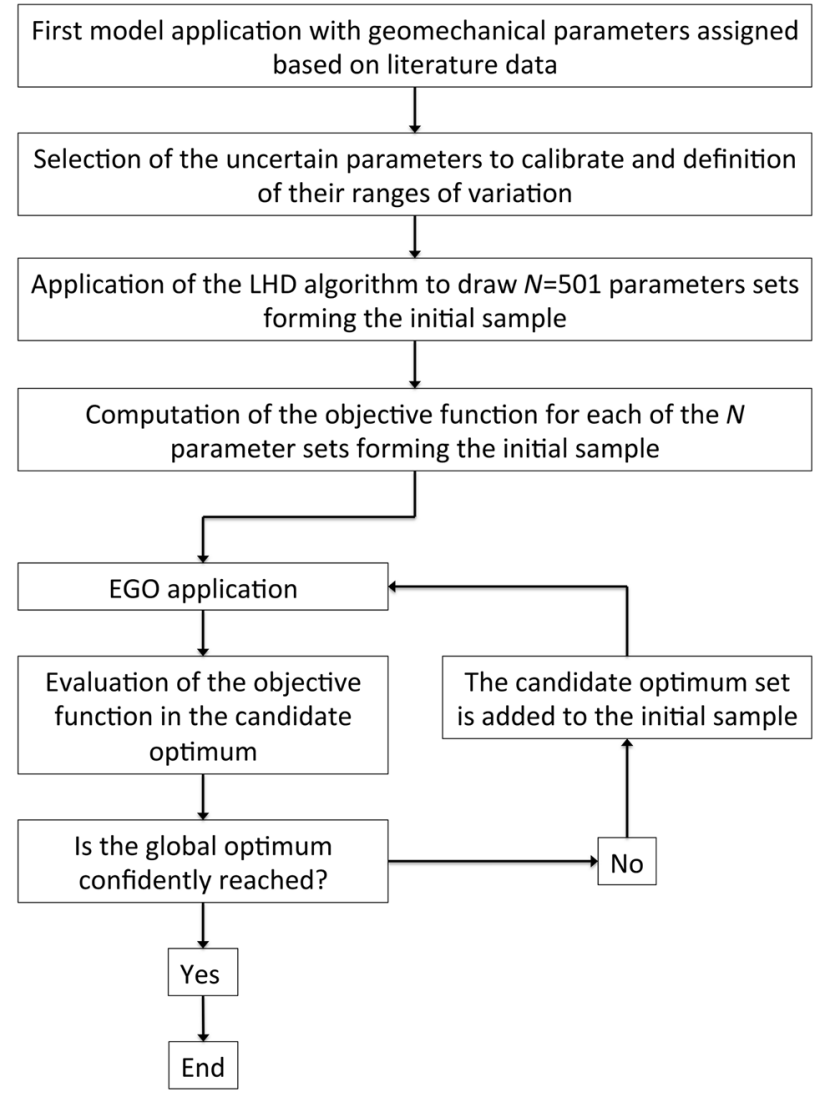

Figure 4. Flowchart illustrating the implemented simulationoptimization procedure. which is a parameter measuring the similarity between the various radar images. An average density of 33 and 50 MPs per $\mathrm{km}^{2}$ is obtained, respectively, for PS and DS, resulting in approximately $150,000 \mathrm{MPs}$, depending on the acquisition geometry. The reference points correspond to PSs characterized by a very stable radar response (temporal coherence $\simeq 1$ ) located outside the area directly affected by the reservoir development. The SqueeSAR outcome from ENVISAT images is shown in Figure 3a. Notice that, due to the small incident angle (approximately $21^{\circ}$ ), the ENVISAT results are sensitive almost exclusively to the vertical component (land subsidence or uplift) of the movement. Because ascending and descending satellite images are available, the easting components of ground movement are computed on the intersection of the two satellite tracks (Figure 3b). The error introduced by neglecting the nonmeasurable north-south component of the displacements is estimated below $10 \%$ and therefore considered acceptable. The measurements highlight a significant subsidence bowl above the Tengiz reservoir with displacement rates up to $-20 \mathrm{~mm} /$ year. As expected, the horizontal movements converge toward the center of the subsidence bowl with west-east displacement rates up to $8-10 \mathrm{~mm} /$ year.

\section{SIMULATION-OPTIMIZATION PROCEDURE}

This section describes how geomechanical modeling, SARderived measurements of land displacement, and EGO calibration are combined to manage and solve the uncertainties affecting our case study. A flowchart of the implemented simulation-optimization procedure is shown in Figure 4.

A first application of the geomechanical model is carried out consistently with the data collected in the literature and discussed in the "Case study" section. In particular, $c_{M}$ is assumed to decrease linearly with depth between the reported bounds, whereas average values of the reported Poisson's ratio are used in the overburden and reservoir. Moreover, the geomechanical response is assumed linear elastic, i.e., not dependent on the stress history. Based on the quantification provided by Dagistanova et al. (2011), the prescribed pore-pressure evolution decreases at a yearly constant rate $\Delta p$ over the period between 1994 and 2007, with $\Delta p=23 \mathrm{bar} /$ year (2.3 $\mathrm{MPa} /$ year) in the central platform and $\Delta p=13 \mathrm{bar} /$ year (1.3 MPa/year) in the outer platform and upper/lower slope.

Afterward, the EGO approach is applied to improve the numerical results of the geomechanical simulation. Following some considerations on the uncertainty of the collected information, we select the set c of $M=5$ calibration parameters $\mathbf{c}=\left\{c_{1}, c_{2}, c_{3}, c_{4}, c_{5}\right\}$ that are applied as correction factors to reservoir compressibility $\left(c_{1}\right)$, overburden compressibility $\left(c_{2}\right)$, Poisson's ratio $\left(c_{3}\right)$, pressure change in the outer platform and upper slope $\left(c_{4}\right)$, and pressure change in the lower slope $\left(c_{5}\right)$. The ranges of variation of these parameters are discussed in the "Results" section and are summarized in Table 1.

The LHD algorithm is applied to draw the starting sample $\mathbf{c}_{N}$ within the bounded parameter space and $N$ geomechanical simulations are carried out for each parameter set. The numerical results are then combined with the SAR-derived measurements to compute the loss function $L\left(\mathbf{c}_{N}\right)$ for the starting sample. Finally, EGO iterations are performed until the algorithm repeatedly investigates only a small neighborhood of the parameter space, confidently containing the global optimum, and no further $L(\mathbf{c})$ reduction is observed.

Clearly, the size $N$ of the starting sample significantly influences the number of iterations that EGO needs to reach a reasonable approximation of the global optimum. In terms of total number 
of model applications, the trade-off suggested by Jones et al. (1998) is $N=10 \times M$ (50 parameter sets in our case). However, the geomechanical simulations for the starting sample can be carried out in parallel and are less time-expensive compared with the sequential EGO applications. Therefore, we assigned a size the relatively large size $N=501$ to the starting sample, to reduce the total number of EGO iterations and the time needed by the whole simulation-optimization procedure.

\section{RESULTS}

Figure 5a shows the yearly displacement rates $\eta_{\mathbf{c}}$ provided by the noncalibrated numerical model. The shape of the computed subsidence bowl is similar to that provided by SqueeSAR (Figure 3a), confirming that the reservoir geometry is sufficiently well reproduced in the model. However, the maximum subsidence rate is largely overestimated, i.e., by a factor almost equal to two. In addition, the computed areal extent of the coastland portion affected by the oil field development is much larger than the monitored evidence. The over- estimate is clearly quantified in Figure $6 \mathrm{a}$, where the error $e_{r r}=\eta_{\mathbf{c}}$ $\eta_{\mathbf{m}}$ above the trace of the reservoir is provided.

We account for the initial overestimation of the computed land subsidence and the information provided by other geomechanical studies in similar geologic settings (Carmichael, 1989) to prescribe proper ranges of variation for the calibration parameters (see Table 1). In particular, the rock compressibility can be reduced up to one order of magnitude, the Poisson's ratio can span the $0.1-$ 0.5 range common to geomaterials (Zoback, 2007), the pressure change in the outer portions of the reservoir can possibly vanish. Once the ranges of variation are assigned, the starting sample $\mathbf{c}_{N}$ of $N=501$ parameter sets is drawn and the loss function $L\left(\mathbf{c}_{N}\right)$ evaluated through parallel model applications.

Afterward, 67 EGO iterations are performed until the method confidently provides the coordinates of the global optimum. Figure 7a shows the frequency distribution of the $L\left(\mathbf{c}_{N}\right)$ values, computed for the starting parameter set $\mathbf{c}_{N}$, whereas Figure $7 \mathrm{~b}$ shows the progressive reduction of the loss function during the EGO iterations. It can be observed that the last 21 EGO iterations correspond

Table 1. Summary of the selected calibration parameters, along with the assigned ranges of variation, EGO outcome, and sequential calibration outcome. The reader is referred to the "Case study" section for a more detailed characterization of the reservoir geometry.

Calibration parameter

\begin{tabular}{ll}
\hline$c_{1}$ & Reservoir compressibility \\
$c_{2}$ & Overburden compressibility \\
$c_{3}$ & Poisson's ratio \\
$c_{4}$ & Pressure variation in lateral platform and upper slope \\
$c_{5}$ & Pressure variation in lower slope
\end{tabular}

Range of variation EGO optimum Sequential optimum

$\begin{array}{lll}0.10-1.00 & 0.43 & 0.80 \\ 0.10-1.00 & 0.10 & 0.90 \\ 0.50-1.40 & 0.50 & 1.00 \\ 0.00-1.00 & 0.54 & 0.00 \\ 0.00-1.00 & 0.00 & 0.00\end{array}$

0.80

0.90

1.00

0.00

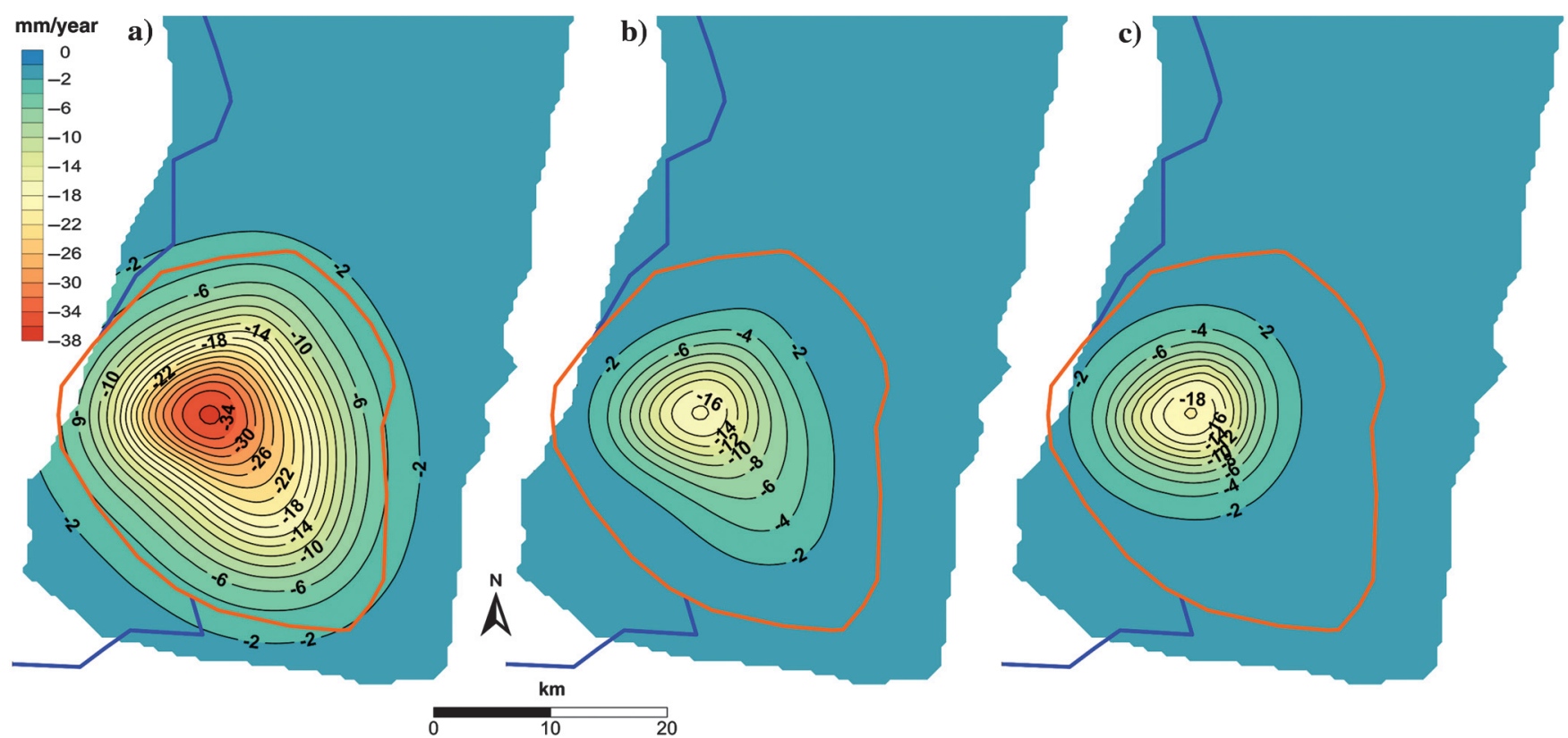

Figure 5. Displacement rates (mm/year) as computed by the FE model (a) using the hydrogeomechanical data set published in the literature, (b) after calibration by EGO, and (c) after sequential optimization of single parameters. Negative values mean land subsidence. The traces of the Tengiz reservoir and the coastline of the Caspian Sea are shown by the orange and the blue lines, respectively. 
to approximately the same loss value, as EGO repeatedly investigates only a small neighborhood around the current optimum. The only two exceptions are runs \#53 and \#58, where EGO tries to sample in poorly explored regions, receiving a negative feedback (see also Figure $8 \mathrm{a}$ and $8 \mathrm{~b}$ ). Although we cannot guarantee that the global optimum is reached, due to the unknown nature of the loss function, we can still argue that the practical convergence condi- tions discussed in the "Efficient global optimization" section are confidently met.

Figure $8 \mathrm{a}$ and $8 \mathrm{~b}$ shows the $c_{1}$ versus $c_{4}$ points corresponding to the starting sample (smaller dots) and to the 67 iterations performed by EGO (larger dots). The dots are colored by iteration in Figure 8a and by loss value in Figure 8b. No configuration far from the location of the optimum configuration is characterized by a loss value
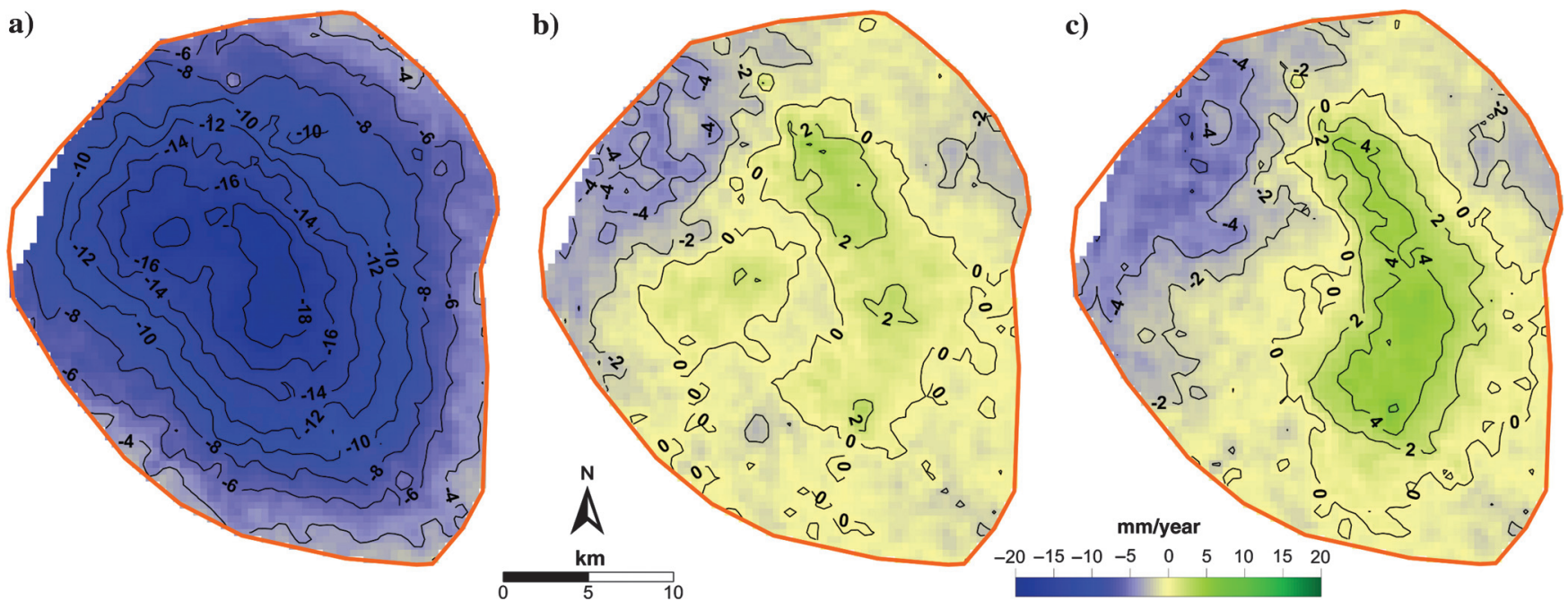

Figure 6. Map of $e_{r r}$ between the SqueeSAR measurements on ENVISAT images and the simulated subsidence above the trace of the Tengiz reservoir with (a) no calibration, (b) calibration via EGO, and (c) calibration via sequential optimization of single parameters. Negative values indicate an overestimate of the anthropogenic land subsidence, whereas positive values indicate an underestimate. The trace of the Tengiz reservoir is shown by the orange line.

Figure 7. (a) Distribution of the loss values corresponding to the 501 simulations of the starting sample $\mathbf{c}_{N}$. (b) Nonmonotonic loss reduction during the 67 EGO iterations (red dots) and minimum loss value obtained at the end of the sequential optimization (blue line). The EGO procedure over performs the sequential optimization at iteration \#23.

Figure 8. Position in the $\left(c_{1}, c_{4}\right)$ plane of the 501 points of the initial subset extracted with a LHD method (small dots) and of the 67 iterations investigated by EGO (large dots). Dots are colored based on the iteration number in panel (a) and $L(\mathbf{c})$ in panel (b). The position of points \#53 and \#58 shows that EGO may predict the maximum expected improvement in regions where the sampling density is lower, even if far from the current optimum. The optimum provided by the sequential optimization is highlighted in panel (b) and lies relatively far from the global optimum. a)

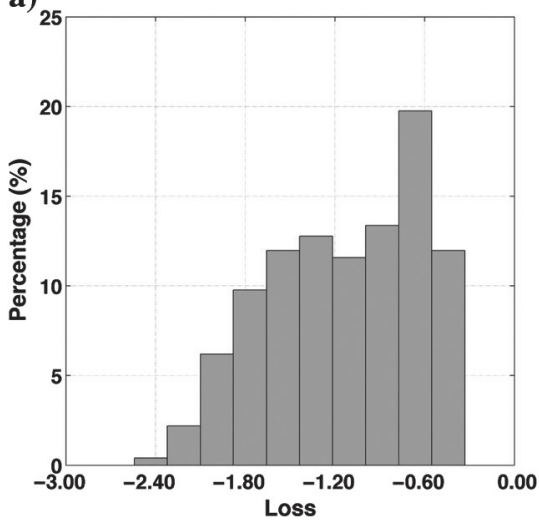

b)

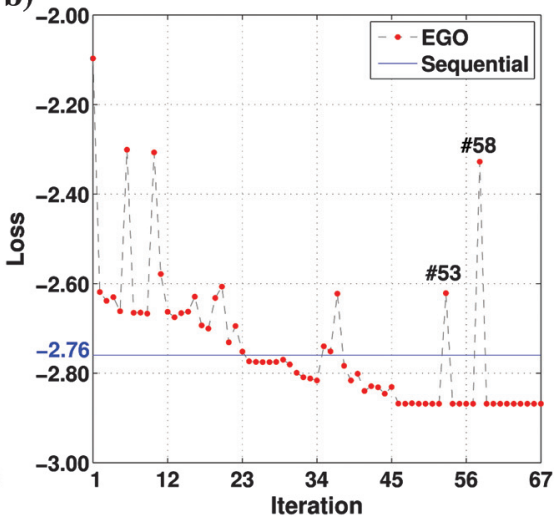

a)

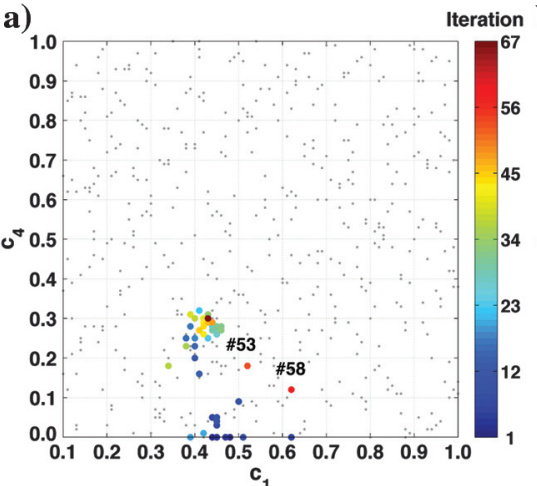

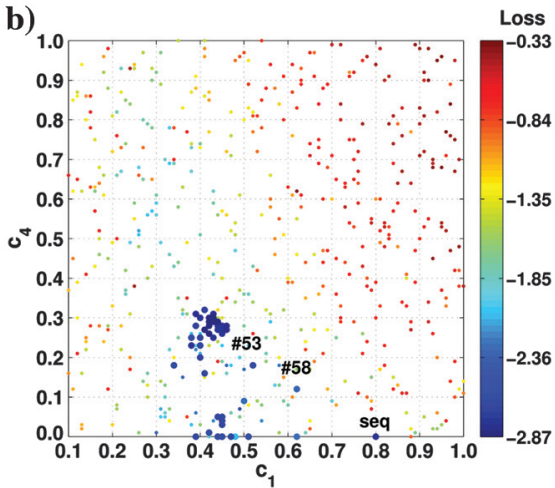


similar to the global minimum, which suggests that the set of $M=5$ selected parameters led to a well-posed problem. The positions of candidate sets \#53 and \#58, highlighted in Figure 8a and 8b, suggest how EGO samples away from the current optimum when the expected value of the loss function is locally balanced by the standard deviation, as discussed in the "Efficient global optimization" section. In a similar way, further EGO iterations would eventually sample all the other unexplored regions of the parameter space.

As expected, an increase of the model performance requires a reduction of the soil compressibility $\left(c_{1}=0.43\right.$ and $\left.c_{2}=0.10\right)$. As far as the Poisson's ratio is concerned, a significant reduction is required as well $\left(c_{3}=0.50\right)$, yielding values that are more consistent with those typical of rock $(0.15-0.25)$ usually characterized by a significant plasticity (see, e.g., Carmichael, 1989). The yearly pressure decreases in the outer platform and the upper slope is almost halved to $7 \mathrm{bar} /$ year $\left(c_{4}=0.54\right)$, whereas a null pressure variation is required in the lower slope $\left(c_{5}=0.0\right)$. These numbers are likely reasonable on account of the high variability of the pressure change due to the fractured nature of the carbonate rocks in the outer reservoir (Dagistanova et al., 2011).

The results obtained by the geomechanical model calibrated via EGO are shown in Figure 5b. The subsidence map is much more adherent to the available measurements with respect to the initial simulation. The function $e_{r r}$ generally decreases to $1-2 \mathrm{~mm} /$ year and peaks at $3-5 \mathrm{~mm} /$ year only in the westernmost portion of the reservoir (Figure 6b), in which the measurements show an uplift unlikely connected to the reservoir production (Figure 3a) and therefore hardly reproducible through simulations.

Sequential calibrations of single parameters were also carried to test whether simple optimization methods can provide solutions
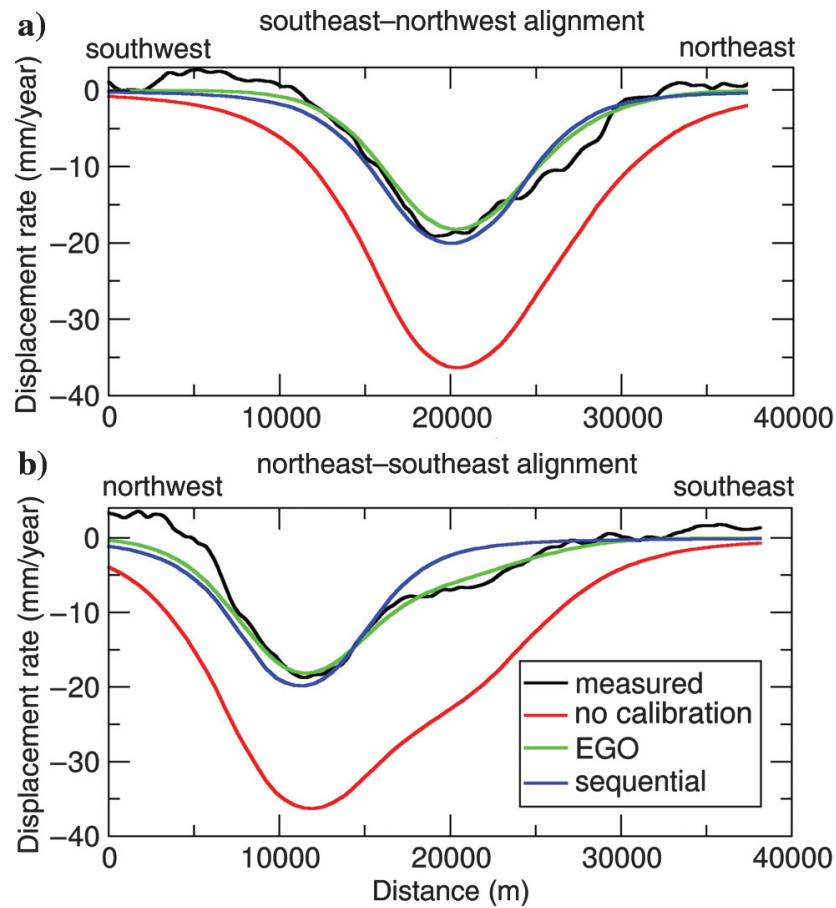

Figure 9. Comparison between the measured (black line) and computed (red line for no calibration, green line for EGO optimization and blue line for sequential optimization) land displacement rates, along (a) the southwest-northeast alignment (shown in Figure 1b) and (b) the perpendicular northwest-southeast alignment. comparable to EGO. Each parameter is calibrated by testing 10 equally spaced values in the corresponding range of variation and selecting the one that yields the smallest loss. A complete sequential calibration requires a total number of $10 \times M=50$ loss function evaluations. The results obtained for different sequences of parameters highlight that the identified optima lie far from the global one and the corresponding loss function values are significantly larger than the global minimum. For illustrative purposes, Figures $5 \mathrm{c}$ and $6 \mathrm{c}$ show the displacement rate and the error maps corresponding to the best of all sequential optimizations (loss $=-2.76$ ), obtained by calibrating, in the order, $c_{4}=0.00$, $c_{5}=0.00, c_{1}=0.80, c_{2}=0.90$, and $c_{3}=1.00$. The location of this optimum is relatively far from the EGO optimum, as highlighted in Figure 8b. Moreover, Figure 7b shows how EGO over performs the sequential optimization in terms of loss reduction already after iteration $\# 23$. These results suggest that simple optimization methods, which can prove effective for problems with few degrees of freedom, become inefficient compared with global optimization ones when the number of calibration parameters increases.

Figure 9 shows a comparison between measured and computed land displacement rates for the various simulations addressed by this study, along the southwest-northeast alignment (see also Figure 1b) and along the perpendicular alignment northwest-southeast. The EGO and the sequential optimization method provide significant improvements with respect to the initial simulation but, as highlighted in Figure $9 b$, the sequential optimization clearly underestimates the subsidence rate along the northeast-southwest alignment.

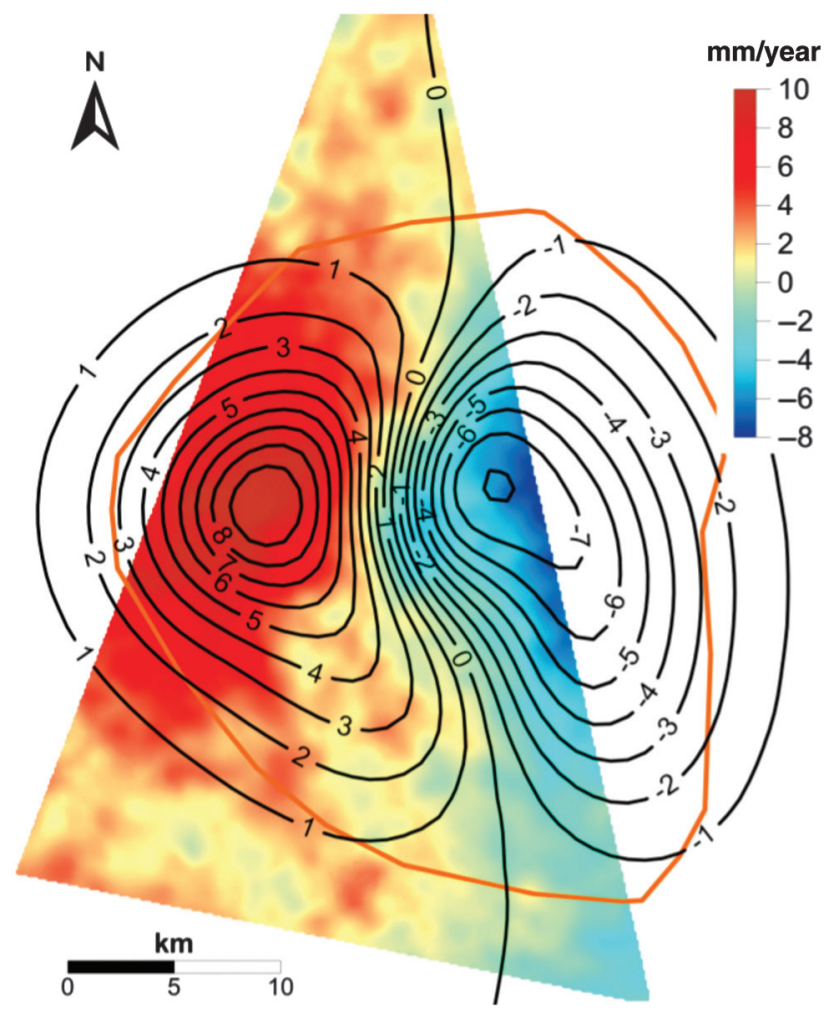

Figure 10. Comparison between the west-east land displacements measured by SqueeSAR (colored map) and those computed by the FE model (black isolines) calibrated via EGO. Positive values mean eastward movement. The trace of the Tengiz reservoir is shown by the orange line. 
Finally, the EGO-calibrated geomechanical model was validated using the measured easting movements. In Figure 10, the west-east displacement rates computed by the EGO-calibrated model are compared with the measurements obtained by combining the SqueeSAR technique on the ENVISAT descending and RADARSAT-1 ascending acquisitions. The figure highlights an excellent agreement between the measurements and model outcome, confirming that the EGO calibration provided a set of physically meaningful parameters.

\section{CONCLUSIONS}

With very few exceptions such as the In Salah field in the Algerian Sahara desert where $\mathrm{BP}$ reinjects the $\mathrm{CO}_{2}$ separated from the produced $\mathrm{CH}_{4}$ and the Lombardia reservoir in northern Italy managed by Stogit for UGS activities, one common problem in the geomechanical characterization of deep reservoirs is the lack of a comprehensive data set. In all these cases, scientific investigations have to cope with large uncertainties. When more than two uncertain parameters are present, arguing the correct values with only physical intuition, experience, or simple optimization procedures is however problematic.

This paper presented the capability of EGO to couple SAR-based measurements and 3D numerical modeling to infer the geomechanical characteristics of the Tengiz reservoir, Kazakhstan. Being a forward method, the choice of the uncertain parameters and the related variability ranges is an important step that must be based on physical considerations. As is usually done when little information is available, the geomechanical parameters were either assumed to vary linearly with depth (compressibility $c_{M}$ ) or to be spatially homogeneous within certain reservoir regions (Poisson's ratio $\nu$ and pressure drop $\Delta p$ ). Although the geomechanical model was not provided with high-resolution data, the EGO optimized parameters remain valid in an average sense. The primary advantage of EGO is the small computational burden. In fact, after running the model for a sparse starting sample of parameter sets, which was required to grossly investigate the model response within the parameter space, EGO detected the optimal calibration with few additional simulations. Such efficiency stems from the capability of the stochastic metamodeling to predict the maximum expected improvement in poorly explored regions of the parameter space, even if far from the current optimum. Accordingly, a large number of uncertain parameters can be managed simultaneously even with a sparse initial sampling. Overall, EGO can prove a valid alternative to inversion and data assimilation methods to account for uncertainties in geomechanical modeling and to provide reliable quantification of reservoir characteristics.

If new and more accurate information on the Tengiz reservoir will become available, EGO can also be applied to resolve the reservoir geometry and the pressure variation with finer spatial detail. Moreover, EGO can be used to optimize more complex geomechanical relationships linking the elastic properties to the stress history or by differentiating more precisely the behavior of the various rock types forming the pre-Caspian basin in the surroundings of the Tengiz reservoir.

\section{ACKNOWLEDGMENTS}

SqueeSAR data for the study site are available from TeleRilevamento Europa S.r.l. (www.treuropa.com). The authors would like to thank M. Bosch and the other anonymous reviewers for their constructive comments.

\section{REFERENCES}

Alassi, H., R. Holt, and M. Landrø, 2010, Relating 4D seismics to reservoir geomechanical changes using a discrete element approach: Geophysical Prospecting, 58, 657-668, doi: 10.1111/j.1365-2478.2009.00859.x.

Baù, D., M. Ferronato, G. Gambolati, P. Teatini, and A. Alzraiee, 2015, Ensemble smoothing of land subsidence measurements for reservoir geomechanical characterization: International Journal for Numerical and Analytical Methods in Geomechanics, 39, 207-228, doi: 10.1002/nag.v39.2.

Biot, M. A., 1941, General theory of three-dimensional consolidation: Journal of Applied Physics, 12, 155-164, doi: 10.1063/1.1712886.

Bull, A. D., 2011, Convergence rates of efficient global optimization algorithms: Journal of Machine Learning Research, 12, 2879-2904.

Carmichael, R. S., 1989, Practical handbook of physical properties of rocks and minerals: CRC Press.

Castelletto, N., G. Gambolati, and P. Teatini, 2013, Geological $\mathrm{CO}_{2}$ sequestration in multi-compartment reservoirs: Geomechanical challenges: Journal of Geophysical Research: Solid Earth, 118, 2417-2428, doi: 10.1002/ jgrb.50180.

Chang, H., Y. Chen, and D. Zhang, 2010, Data assimilation of coupled fluid flow and geomechanics using the ensemble Kalman filter: SPE Journal, 15, 382-394, doi: 10.2118/118963-PA.

Chang, C., E. Mallman, and M. Zoback, 2014, Time-dependent subsidence associated with drainage-induced compaction in Gulf of Mexico shales bounding a severely depleted gas reservoir: AAPG Bulletin, 98, 11451159, doi: $10.1306 / 11111313009$.

Collins, J. F., J. A. M. Kenter, P. M. Harris, G. Kuanysheva, D. J. Fischer, and K. L. Steffen, 2006, Facies and reservoir-quality variations in the late Visean to Bashkirian outer platform, rim, and flank of the Tengiz buildup, Precaspian Basin, Kazakhstan, in P. M. Harris, and L. J. Weber, eds., Giant hydrocarbon reservoirs of the world: From rocks to reservoir characterization and modeling: AAPG Memoir 88/SEPM Special Publication, 55-95.

Dagistanova, K., A. Aitzhanov, D. Belanger, P. Bateman, R. Camerlo, R. Fitzmorris, M. Hui, G. Jacobs, G. King, C. Laidlaw, W. Narr, Y. Pan, W. Peake, M. Shook, M. Skalinski, M. Sullivan, T. Tankersley, D. Tolessin, A. Yessaliyeva, and A. Zhumagulova, 2011, Integration of dynamic data into characterization of the Tengiz reservoir: Tengiz slope: AAPG European Region Annual Conference, AAPG Search and Discovery Article \#50399.

Ferretti, A., A. Fumagalli, F. Novali, C. Prati, F. Rocca, and A. Rucci, 2011, A new algorithm for processing interferometric data-stacks: SqueeSAR: IEEE Transactions on Geoscience and Remote Sensing, 49, 3460-3470, doi: 10.1109/TGRS.2011.2124465.

Ferronato, M., G. Gambolati, P. Teatini, and D. Baù, 2003, Interpretation of radioactive marker measurements in the northern Adriatic gas fields: SPE Reservoir Evaluation and Engineering, 6, 401-411, doi: 10.2118/79470-PA.

Fielding, E. J., R. G. Blom, and R. M. Goldstein, 1998, Rapid subsidence over oil fields measured by SAR interferometry: Geophysical Research Letters, 25, 3215-3218, doi: 10.1029/98GL52260.

Gambolati, G., M. Ferronato, P. Teatini, R. Deidda, and G. Lecca, 2001, Finite element analysis of land subsidence above depleted reservoirs with pore pressure gradient and total stress formulations: International Journal for Numerical and Analytical Methods in Geomechanics, 25, 307-327, doi: 10.1002/(ISSN)1096-9853.

Hesse, M. A., and G. Stadler, 2014, Joint inversion in coupled quasi-static poroelasticity: Journal of Geophysical Research: Solid Earth, 119, 14251445, doi: 10.1002/2013JB010272.

Hooper, A., 2008, A multi-temporal InSAR method incorporating both persistent scatterer and small baseline approaches: Geophysical Research Letters, 35, L16302, doi: 10.1029/2008GL034654.

Iglesias, M. A., and D. McLaughlin, 2012, Data inversion in coupled subsurface flow and geomechanics models: Inverse Problems, 28, 115009, doi: 10.1088/0266-5611/28/11/115009.

Janna, C., N. Castelletto, M. Ferronato, G. Gambolati, and P. Teatini, 2012, A geomechanical transversely isotropic model of the Po River basin using PSInSAR derived horizontal displacement: International Journal of Rock Mechanics and Mining Sciences, 51, 105-118, doi: 10.1016/j. ijrmms.2012.01.015.

Janna, C., M. Ferronato, and G. Gambolati, 2010, A block FSAI-ILU parallel preconditioner for symmetric positive definite linear systems: SIAM Journal on Scientific Computing, 32, 2468-2484, doi: 10.1137/090779760.

Jones, D. R., 2001, A taxonomy of global optimization methods based on response surfaces: Journal of Global Optimization, 21, 345-383, doi: 10 1023/A:1012771025575.

Jones, D. R., M. Schonlau, and W. J. Welch, 1998, Efficient global optimization of expensive black-box functions: Journal of Global Optimization, 13, 455-492, doi: 10.1023/A:1008306431147.

Kleijnen, J. P., W. van Beers, and I. Van Nieuwenhuyse, 2012, Expected improvement in efficient global optimization through bootstrapped kriging: Journal of Global Optimization, 54, 59-73, doi: 10.1007/ s10898-011-9741-y. 
Lei, X., S. Ma, W. Chen, C. Pang, J. Zeng, and B. Jiang, 2013, A detailed view of the injection-induced seismicity in a natural gas reservoir in Zigong, southwestern Sichuan Basin, China: Journal of Geophysical Research: Solid Earth, 118, 4296-4311, doi: 10.1002/jgrb.50310.

Levasseur, S., Y. Malécot, M. Boulon, and E. Flavigny, 2008, Soil parameter identification using a genetic algorithm: International Journal for Numerical and Analytical Methods in Geomechanics, 32, 189-213, doi: 10.1002/ (ISSN)1096-9853.

Li, Y., J. Yang, and X. Hu, 2000, Origin of ground fissures in the Shanxi Graben system, Northern China: Engineering Geology, 55, 267-275, doi: 10.1016/S0013-7952(99)00082-4.

Lovison, A., and E. Rigoni, 2010, Extracting optimal datasets for metamodelling and perspectives for incremental samplings: Mathematics and Computers in Simulation, 81, 681-692, doi: 10.1016/j.matcom.2010.03.007.

Maxwell, S., J. Rutledge, R. Jones, and M. Fehler, 2010, Petroleum reservoir characterization using downhole microseismic monitoring: Geophysics, 75, no. 5, 75A129-75A137, doi: 10.1190/1.3477966.

Morton, R. A., J. C. Bernier, and J. A. Barras, 2006, Evidence of regional subsidence and associated interior wetland loss induced by hydrocarbon production, Gulf Coast region, USA: Environmental Geology, 50, 261274, doi: 10.1007/s00254-006-0207-3.

Motagh, M., T. R. Walter, M. A. Sharifi, E. Fielding, A. Schenk, J. Anderssohn, and J. Zschau, 2008, Land subsidence in Iran caused by widespread water reservoir overexploitation: Geophysical Research Letters, 35, L16403, doi: 10.1029/2008GL033814.

Oliver, D. S., and Y. Chen, 2011, Recent progress on reservoir history matching: A review: Computational Geosciences, 15, 185-221, doi: 10 $.1007 / \mathrm{s} 10596-010-9194-2$.

Oreste, P., 2005, Back-analysis techniques for the improvement of the understanding of rock in underground constructions: Tunnelling and Underground Space Technology, 20, 7-21, doi: 10.1016/j.tust.2004.04.002.

Rinaldi, A. P., and J. Rutqvist, 2013, Modeling of deep fracture zone opening and transient ground surface uplift at $\mathrm{KB}-502 \mathrm{CO}_{2}$ injection well, In Salah, Algeria: International Journal of Greenhouse Gas Control, 12, 155167, doi: 10.1016/j.ijggc.2012.10.017.
Teatini, P., D. Baú, and G. Gambolati, 2000, Water-gas dynamics and coastal land subsidence over Chioggia Mare field, Northern Adriatic Sea: Hydrogeology Journal, 8, 462-479, doi: 10.1007/s100400000092.

Teatini, P., N. Castelletto, M. Ferronato, G. Gambolati, C. Janna, E. Cairo, D. Marzorati, D. Colombo, A. Ferretti, A. Bagliani, and F. Bottazzi, 2011, Geomechanical response to seasonal gas storage in depleted reservoirs: A case study in the Po River basin, Italy: Journal of Geophysical Research: Earth Surface, 116, F02002, doi: 10.1029/2010JF001793.

Trugman, D. T., A. A. Borsa, and D. T. Sandwell, 2014, Did stresses from the Cerro Prieto Geothermal Field influence the El Mayor-Cucapah rupture sequence?: Geophysical Research Letters, 41, 8767-8774, doi: 10 $.1002 / 2014$ GL061959.

Vasco, D., A. Rucci, A. Ferretti, F. Novali, R. Bissell, P. Ringrose, A. Mathieson, and I. Wright, 2010, Satellite-based measurements of surface deformation reveal fluid flow associated with the geological storage of carbon dioxide: Geophysical Research Letters, 37, L03303, doi: 10 1029/2009GL041544.

Wang, G. G., and S. Shan, 2007, Review of metamodeling techniques in support of engineering design optimization: Journal of Mechanical Design, 129, 370-380, doi: 10.1115/1.2429697.

Weber, L. J., B. P. Francis, P. M. Harris, and M. Clark, 2003, Stratigraphy, lithofacies, and reservoir distribution, Tengiz field, Kazakhstan, in W. M. Ahr, P. M. Harris, W. A. Morgan, and I. D. Somerville, eds., Permocarboniferous carbonate platforms and reefs: SEPM Special Publication 78 and AAPG Memoir 83, 351-394.

$\mathrm{Xu}$, H., J. Dvorkin, and A. Nur, 2001, Linking oil production to surface subsidence from satellite radar interferometry: Geophysical Research Letters, 28, 1307-1310, doi: 10.1029/2000GL012483.

Zienkiewicz, O. C., and R. L. Taylor, 2000, The finite element method (5th ed.): Butterworth-Heinemann.

Zoback, D., 2007, Reservoir geomechanics: Cambridge University Press.

Zoccarato, C., D. Baù, M. Ferronato, G. Gambolati, A. Alzraiee, and P. Teatini, 2016, Data assimilation of surface displacements to improve geomechanical parameters of gas storage reservoirs: Journal of Geophysical Research: Solid Earth, 121, doi: 10.1002/2015JB012090. 